\title{
openheart Anatomical predictors of conduction damage after transcatheter implantation of the aortic valve
}

\author{
Justin T Tretter, ${ }^{\oplus 1,2}$ Shumpei Mori, ${ }^{3}$ Robert H Anderson, ${ }^{4}$ Michael D Taylor, ${ }^{1,2}$ \\ Nicholas Ollberding, ${ }^{2,5}$ Vien Truong, ${ }^{6}$ Joseph Choo, ${ }^{6}$ Dean Kereiakes, ${ }^{6}$ \\ Wojciech Mazur ${ }^{6}$
}

To cite: Tretter JT, Mori S, Anderson RH, et al. Anatomical predictors of conduction damage after transcatheter implantation of the aortic valve. Open Heart 2019;6:e000972. doi:10.1136/ openhrt-2018-000972

Received 19 November 2018 Revised 20 February 2019 Accepted 4 March 2019

Check for updates

(C) Author(s) (or their employer(s)) 2019. Re-use permitted under CC BY-NC. No commercial re-use. See rights and permissions. Published by BMJ.

${ }^{1}$ Heart Institute, Cincinnati Children's Hospital Medical Center, Cincinnati, Ohio, USA ${ }^{2}$ Department of Pediatrics, University of Cincinnati College of Medicine, Cincinnati, Ohio, USA

${ }^{3}$ Division of Cardiovascular Medicine, Department of Internal Medicine, Kobe University Graduate School of Medicine, Kobe, Japan

${ }^{4}$ Institute of Genetic Medicine Newcastle University, Newcastle-upon-Tyne, UK ${ }^{5}$ Division of Biostatistics and Epidemiology, Cincinnati Children's Hospital Medical Center, Cincinnati, Ohio, USA ${ }^{6}$ Department of Ohio Heart and Vascular, The Christ Hospital and Lindner Center for Research and Education, Cincinnati, Ohio, USA

Correspondence to Dr Justin T Tretter; justin. tretter@cchmc.org

\section{ABSTRACT}

Objective Conduction damage following transcatheter aortic valve implantation (TAVI) remains common. Anatomical risk factors remain elusive. We assessed the impact of variability in the dimensions of the membranous septum and position of the aortic root on the occurrence of conduction damage following TAVI.

Methods The dimensions of the membranous septum, the rotational position of the aortic root correlating to variability in the central fibrous body width, and wedging of the aortic root were assessed on pre-TAVI CT datasets. The depth of implantation was measured from the final aortic angiogram. The variables were compared with the occurrence of both permanent pacemaker insertion (PPI) and left bundle branch block (LBBB) following TAVI.

Results of 200 patients who met inclusion criteria (mean age $=81$ years $\pm 7.7,49 \%$ men), $20.5 \%$ underwent PPI after TAVI. New LBBB occurred in $23.5 \%, 21.3 \%$ of whom required $\mathrm{PPI}$. Preprocedural right bundle branch block $(\mathrm{OR}$ $=7.00 ; \mathrm{Cl} 3.13$ to 15.64$)$, valve type $(\mathrm{OR}=2.35 ; \mathrm{Cl} 1.13$ to 4.87), depth of implantation ( $\mathrm{OR}=1.62$; $\mathrm{Cl} 1.01$ to 2.61) and the difference between depth of implantation and the distance from the virtual basal ring to the inferior margin of the membranous septum $(\mathrm{OR}=0.61 ; \mathrm{Cl} 0.38$ to 0.99$)$ were all associated with $\mathrm{PPI}$, with similar associations with LBBB. No gross anatomical variable alone was associated with conduction damage.

Conclusions Gross anatomical variation of the aortic root and its underlying support, including the membranous septum, were not associated with the occurrence of either PPI or new LBBB. Procedural characteristics associated with these adverse outcomes suggest that the depth of implantation and radial force of the bioprosthesis, regardless of gross anatomical variability, increase the risk for conduction damage.

\section{INTRODUCTION}

Conduction damage and the need for permanent pacemaker implantation (PPI) remain common following transcatheter aortic valve implantation (TAVI). Despite a reduction in the general rates of PPI following TAVI with newer-generation valves, ${ }^{1}$ the rates remain relatively high, ${ }^{12}$ with some studies reporting an increased incidence with

\section{Key messages}

What is already known about this subject?

- Crude measurements of the height of the membranous septum suggest a correlation to conduction damage following transcatheter aortic valve implantation (TAVI). However, the distance from the plane of the virtual basal aortic ring to the crest of the muscular septum (the presumed location of atrioventricular conduction axis) averages $2-3 \mathrm{~mm}$, and is impinged much more frequently by aortic valve prostheses than is reflected by the incidence of permanent pacemaker insertion (PPI) or new left bundle branch block (LBBB) following TAVI.

What does this study add?

- Gross anatomical variation of the aortic root and its underlying support, including the dimensions of the membranous septum and the rotational position correlating to variability in the width of the central fibrous body, are not associated with the occurrence of either PPI or new LBBB following TAVI. TAVI valve type and depth of implantation are the primary procedural associations with conduction damage regardless of gross anatomical variation.

How might this impact on clinical practice?

- This observation may impact selection of the type of valve as well as its planned depth of implantation, and should inform future anatomical investigations in TAVI.

newer-generation valves. ${ }^{2}$ This is of particular concern with respect to the extension of this therapy to younger patients deemed to be at lower risk. ${ }^{3-5}$ CT assessment prior to implantation is the standard of care for procedural planning. ${ }^{6}$ Studies assessing risk for conduction damage have pointed to the importance of the height of the membranous septum serving as a surrogate for the position of the atrioventricular conduction axis relative to the aortic root. In this regard, two recent studies, using a standard coronal plane, reported that the 'length' of the membranous septum, better termed its height, is a 
predictor for conduction damage following placement of either balloon-expandable ${ }^{7}$ or self-expandable valves, ${ }^{8}$ especially when compared with the depth of their implantation. $^{78}$

Previously reported methods used for assessing the height of the membranous septum are anatomically crude, measuring obliquely across this structure, including a variable portion of the overlying interleaflet triangle in the recorded dimension. ${ }^{9}$ The clinically relevant measurement is that between the plane of the virtual basal ring and the inferior margin of the membranous septum, which we will describe as the 'inferior distance'. This corresponds to the estimated distance between the virtual basal ring plane and the atrioventricular conduction axis. It often measures only a portion of the height of the membranous septum, as there is considerable variation in the position of the membranous septum relative to the plane of the basal ring. ${ }^{10}$ This measurement is much shorter than expected, ${ }^{11}$ indicating that the inferior margin of the membranous septum and its junction with the crest of the muscular septum is likely impinged by the prosthesis in up to $90 \%$ of insertions. Considering the lesser frequency of PPI, ${ }^{2}$ other variables are likely involved in producing conduction system damage. The impact of the dimensions of the membranous septum on conduction damage and the requirement for PPI, along with its inferior distance, when measured using an anatomically accurate method, remain unknown. ${ }^{10} 11$ Other variations in aortic root morphology and its underlying support, including its wedging and rotational position, ${ }^{11} 12$ could influence the risk of PPI following TAVI. ${ }^{11}$

We aimed to determine the impact of the dimensions of the membranous septum, including its inferior distance, and additional anatomical variables when measured using anatomically accurate methods, on PPI requirement and the development of complete left bundle branch block (LBBB) following TAVI.

\section{METHODS}

\section{Study population}

We analysed retrospectively CT angiographic datasets from 381 consecutive patients undergoing TAVI from December 2013 to December 2017 at The Christ Hospital in Cincinnati, Ohio. From this cohort, we excluded 181 patients. Of those excluded, 97 patients had undergone CT angiography using decreased contrast volume injected via the pulmonary arteries. This protocol is used for those with serum creatinine greater than 1.6, as previously described by our group. ${ }^{13}$ The technique limits the quality necessary for accurate assessment of the membranous septum dimensions. A further 61 patients had undergone PPI prior to TAVI, 12 patients already had surgically inserted bioprosthetic valves and 11 patients were known to have congenitally bicuspid valves. The study involving the remaining 200 patients was approved by the institutional review board (IRB) of both the Christ Hospital Health Network and Cincinnati Children's
Hospital Medical Center. The need for informed consent was waived per both IRBs.

\section{Baseline demographics, clinical characteristics and procedural data}

Baseline preprocedural demographic and clinical information was recorded, including baseline left ventricular ejection fraction obtained on preprocedural transthoracic echocardiogram and comorbidities used to calculate the Society of Thoracic Surgery (STS) mortality risk score. Procedural information recorded included the type and size of valve deployed during the procedure, as well as the preprocedural and postprocedural rhythm and presence of bundle branch block prior to TAVI. Primary outcomes were the occurrence of PPI or new-onset LBBB following the procedure. Given that approximately $90 \%$ receiving PPI following TAVI will require this within 1 week after the procedure, and the majority of the remaining $10 \%$ will require this within 1 month after the procedure, ${ }^{14}$ we reviewed all records for up to at least 6 months following the procedure.

\section{Protocol for CT angiography}

All patients underwent electrocardiographically gated cardiac CT angiography, without dose modulation, using the Philips Brilliance iCT 256-slice CT scanner, and $80-120 \mathrm{~mL}$ of total intravenous contrast. Images were reconstructed at $35 \%, 40 \%, 45 \%$ and $75 \%$ of the R-R interval, with a slice thickness of $0.9 \mathrm{~mm}$ and 0.45 $\mathrm{mm}$ spacing. The tube voltage was $120 \mathrm{kV}$, and automatic current modulation was used. All subsequent processing was performed on the Vitrea workstation. Using multiplanar orthogonal reformatting, we measured the location of the virtual basal ring, the extent of rotation and wedging of the aortic root and the dimensions of the membranous septum at $40 \%$ of the R-R interval.

\section{CT and fluoroscopic angiographic measurements}

The location of the so-called aortic annulus, better termed the virtual basal ring, which serves as the target structure for sizing of the inserted valve, was determined by joining together the nadirs of attachment of the semilunar hinges of the valvar leaflets, as previously described. ${ }^{6}$ This permitted the calculation of its longest and shortest diameters, its area and its circumference. We then calculated an area-derived diameter, which was compared with the diameter of the inserted valve to show the extent of valvar oversizing. ${ }^{15}$

The height and width of the membranous septum, as well as the distance between the virtual basal ring plane and the junction of the inferior margin of the membranous septum with the crest of the muscular ventricular septum, which we describe as the inferior distance, were measured using our previously validated methods (figure 1). ${ }^{1011}$ We then assessed the angle of tilting of the aortic root in the frontal and reformatted threechamber planes. ${ }^{10}$ The extent of wedging and rotation of the root were measured according to previously validated 

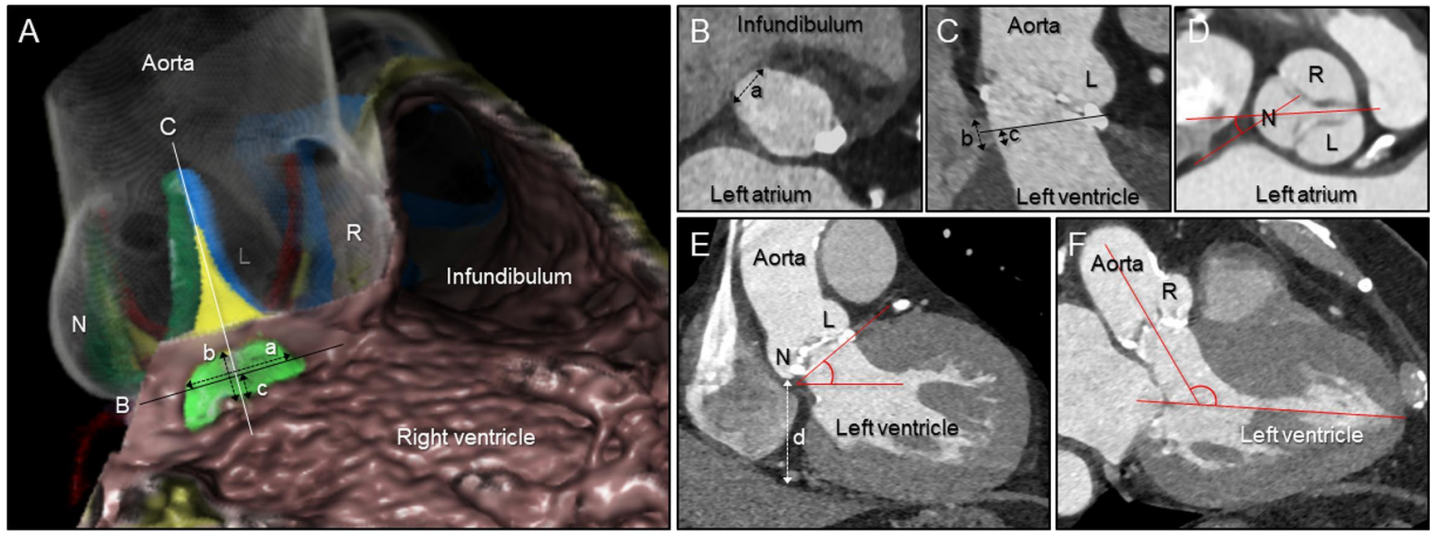

Figure 1 Measurements using multiplanar reconstruction images. (A) Virtual dissection images of the aortic root and membranous septum viewed from the right anterior oblique and caudal direction, showing the concept of the measurements. Actual measurements were all performed using multiplanar reconstruction images (B-F). The membranous septum (yellowgreen area) is located inferior to the interleaflet triangle (yellow area) between the right $(\mathrm{R})$ and non-coronary $(\mathrm{N})$ aortic sinuses, interposed by the inner heart curvature. The black line (B) corresponds to the plane of the virtual basal ring represented in panel B. The white line corresponds to the orthogonal plane bisecting the interleaflet triangle between the $\mathrm{R}$ and $\mathrm{N}$, as shown in panel C. (B) The width of the membranous septum (a) was determined from the plane of the virtual basal ring. (C) The height of the membranous septum (b) was measured from the crest of the muscular ventricular septum and the inner heart curvature. The inferior distance (c) was determined from the plane of the virtual basal ring (black line) to the crest of the muscular ventricular septum. (D) The angle of rotation of the root was defined as the angle between the line bisecting the right fibrous trigone and the line bisecting the $\mathrm{N}$ and interleaflet triangle between both $\mathrm{R}$ and left $(\mathrm{L})$ coronary aortic sinuses, measured at the level of the sinus of Valsalva. (E) Using the standard coronal section, the wedged height (d) was defined as the vertical distance between the lowest point of the $\mathrm{N}$ and the inferior cardiac epicardial border. Aortic tilting angle was also determined. (F) We measured the angulation of the aortic root relative to the left ventricle using the reformatted three-chamber plane.

methods. ${ }^{11} 12$ The previously demonstrated correlation between deeper wedging and a shorter inferior distance ${ }^{11}$ implies that patients with deeper wedging should have greater likelihood of more extensive impingement of the prosthetic valve below the plane of the basal ring, hence potentiating injury to the atrioventricular conduction axis and left bundle branch. We also showed that the central fibrous body, and related fibrous support of the aortic root, is broader when the aortic root is positioned in clockwise fashion compared with a central position, and even more so when compared with the counterclockwise positioned aortic root. It is unclear whether this variation increases the length of the atrioventricular conduction axis coursing immediately adjacent to the clockwise positioned root and, thus the potential risk of conduction damage. ${ }^{11}$ The depth of the implanted valve was assessed from the final fluoroscopic aortic angiogram acquired after deployment, using the distance from the nadir of the non-coronary sinus to the lower edge of the frame of the valve stent. The inferior distance measurement of the membranous septum was subtracted from the depth of valve implantation ( $\Delta$ inferior distance to implantation depth).

\section{Statistical analysis}

Means and SD, and frequencies and percentages, were used to describe procedural, demographic and clinical characteristics. Independent samples t-tests and Fisher's exact tests were used to test for differences in baseline preprocedural demographic and clinical characteristics. ORs and 95\% CIs for PPI and LBBB following TAVI were obtained via univariate logistic regression using the $1 \mathrm{rm}$ function in the rms package.$^{16}$ Given the variation in the units of measurement, ORs for a unit increase equal to the IQR are presented for all continuous variables to aid interpretation. Potential non-linear associations were examined using restricted cubic splines. These were retained where the Wald test for the joint non-linear model terms was $p<0.05$. Multivariable models were fitted to obtain adjusted ORs for selected measures. Multicollinearity was assessed using the variance inflation factor and did not exceed 2.6 in any model. Sensitivity analyses were conducted restricting analyses to patients with balloon- or self-expanding valves and to those with no history of atrial fibrillation at baseline. Pearson correlations were used to describe associations between procedural characteristics. All statistical analyses were conducted using R V.3.5.0. ${ }^{17}$

\section{RESULTS \\ Baseline demographics, clinical characteristics and procedural data}

The baseline preprocedural demographic and clinical characteristics for the 200 patients are shown in table 1 . The mean age of the patients was 81 years $\pm 7.7,49 \%$ being men, with a mean body mass index of $28.4 \pm 6 \mathrm{~kg} / \mathrm{m}^{2}$. A permanent pacemaker had been inserted in 41 patients $(20.5 \%)$, all within the first month and 40 within the first week following the procedure. This was because of high-degree atrioventricular block in 26 patients $(63.4 \%)$, symptomatic bradycardia with lesser degree heart block associated with LBBB in 9 (22\%) and symptomatic bradycardia in the 


\begin{tabular}{|c|c|c|c|c|}
\hline & All patients & PPI & No PPI & \\
\hline & $(\mathrm{n}=200)$ & $(n=41)$ & $(n=159)$ & $P$ value \\
\hline Age (years), mean (SD) & $81.0(7.7)$ & $82.1(7.3)$ & $80.7(7.8)$ & 0.29 \\
\hline Male, n (\%) & $98(49.0)$ & $21(51.2)$ & $77(48.4)$ & 0.86 \\
\hline Body mass index $\left(\mathrm{kg} / \mathrm{m}^{2}\right)$, mean (SD) & $28.4(6.0)$ & $28.3(5.7)$ & $28.4(6.1)$ & 0.93 \\
\hline Height (cm), mean (SD) & $167.6(10.3)$ & $168.6(9.4)$ & $167.4(10.5)$ & 0.47 \\
\hline Weight $(\mathrm{kg})$, mean (SD) & $79.9(18.5)$ & $80.8(18.5)$ & $79.7(18.6)$ & 0.73 \\
\hline Creatinine (mg/dL), mean (SD) & $1.05(0.84)$ & $1.02(0.58)$ & $1.05(0.9)$ & 0.81 \\
\hline Left ventricle EF (\%), mean (SD) & $55.7(11.5)$ & $57.5(10.1)$ & $55.3(11.8)$ & 0.24 \\
\hline QRS duration (ms), mean (SD) & $108.7(24.0)$ & $115.7(26.2)$ & $106.9(23.1)$ & 0.05 \\
\hline RBBB, n (\%) & $34(17.0)$ & $18(43.9)$ & $16(10.1)$ & $<0.01$ \\
\hline LBBB, n (\%) & $14(7.0)$ & $1(2.4)$ & $13(8.2)$ & 0.31 \\
\hline Atrial fibrillation, $\mathrm{n}(\%)$ & $61(30.5)$ & $13(31.7)$ & $48(30.2)$ & 0.85 \\
\hline Coronary artery disease, $\mathrm{n}(\%)$ & $133(66.5)$ & $26(63.4)$ & $107(67.3)$ & 0.71 \\
\hline Chronic obstructive pulmonary disease, $\mathrm{n}(\%)$ & $42(21.0)$ & $8(19.5)$ & $34(21.4)$ & 0.99 \\
\hline Cerebral vascular accident, $\mathrm{n}(\%)$ & $17(8.5)$ & $7(17.1)$ & $10(6.3)$ & 0.05 \\
\hline Diabetes mellitus, $n(\%)$ & $62(31.0)$ & $15(36.6)$ & $47(29.6)$ & 0.45 \\
\hline Hypertension, $n(\%)$ & $179(89.5)$ & $38(92.7)$ & $141(88.7)$ & 0.58 \\
\hline Peripheral vascular disease, $\mathrm{n}(\%)$ & $38(19.0)$ & $11(26.8)$ & $27(17.0)$ & 0.18 \\
\hline Renal disease, $\mathrm{n}(\%)$ & $58(29.0)$ & $14(34.1)$ & $44(27.7)$ & 0.44 \\
\hline The Society of Thoracic Surgery Risk Score, mean (SD) & $4.7(2.8)$ & $5.2(3.4)$ & $4.6(2.7)$ & 0.26 \\
\hline
\end{tabular}

$P$ value from independent samples t-test for continuous variables and Fisher's exact test for categorical variables.

EF, ejection fraction; LBBB, left bundle branch block; PPI, permanent pacemaker insertion; RBBB, right bundle branch block; TAVI,

transcatheter aortic valve implantation.

remaining 6 (14.6\%). New LBBB without high-degree atrioventricular block occurred in 47 patients $(23.5 \%)$. Of those with new LBBB, 10 (21.3\%) had PPI. No preprocedural demographics showed a statistically significant association with PPI. Presence of right bundle branch block (RBBB) prior to the procedure (PPI $43.9 \%$ vs no PPI $10.1 \%$, $\mathrm{p}<0.01$; $\mathrm{OR}=7.00$; CI 3.13 to 15.64 ) was the only preprocedural clinical characteristic associated with PPI.

Implanted valve characteristics and their association with both PPI and new-onset LBBB following TAVI are reported in tables 2-4. A balloon-expanding valve (Sapien XT or Sapien 3, Edwards Lifesciences, Irvine, CA, USA) was used in 94 patients $(47 \%)$ while a mechanically or self-expanding valve was used in the remaining 106 patients (53\%). Of the latter group, 30 (28.3\%) received the mechanically expanding LOTUS valve (Boston Scientific, Natick, MA, USA) while the remaining 76 patients (71.7\%) received the self-expanding CoreValve, Evolut R or Evolut Pro valve (Medtronic, Minneapolis, MN, USA). PPI occurred more frequently following insertion of

Table 2 CT and procedural characteristics according to PPI following TAVI

\begin{tabular}{llll}
\hline & All patients & PPI & No PPI \\
\hline & $(\mathrm{n}=200)$ & $(\mathrm{n}=41)$ & $159)$ \\
\hline Aortic root rotation $\left(^{\circ}\right)$ & $11.2(14.5)$ & $10.4(14.0)$ & $11.4(14.6)$ \\
\hline Frontal aortic root tilt angle $\left(^{\circ}\right)$ & $48.9(9.8)$ & $49.0(9.3)$ & $48.9(9.9)$ \\
\hline Left ventricle to aortic root angle: three-chamber plane $\left(^{\circ}\right)$ & $128.1(8.5)$ & $127.3(8.9)$ & $128.3(8.4)$ \\
Membranous septum height $(\mathrm{mm})$ & $4.3(1.7)$ & $4.2(1.4)$ & $4.3(1.7)$ \\
Inferior distance $(\mathrm{mm})$ & $3.0(2.1)$ & $2.8(1.9)$ & $3.1(2.1)$ \\
Valve implantation depth $(\mathrm{mm})$ & $5.7(2.6)$ & $6.4(2.9)$ & $5.5(2.5)$ \\
\hline Inferior distance to implantation depth $(\mathrm{mm})$ & $-2.7(3.3)$ & $-3.6(3.4)$ & $-2.5(3.2)$ \\
\hline
\end{tabular}

Values are mean (SD).

$\mathrm{PPI}$, permanent pacemaker insertion; TAVI, transcatheter aortic valve implantation; $\Delta$ Inferior distance to implantation depth, difference between inferior distance and valve implantation depth; Inferior distance, virtual basal ring to muscular ventricular septum height. 
Table $3 \mathrm{CT}$ and procedural characteristics and their association with permanent pacemaker insertion following TAVI

\begin{tabular}{|c|c|c|c|c|}
\hline & Q1/reference & Q3/risk & OR (95\% Cl) & $P$ value \\
\hline Aortic root rotation $\left({ }^{\circ}\right)$ & 2.05 & 19.25 & $0.92(0.61$ to 1.39$)$ & 0.69 \\
\hline Aortic root qualification & central/counterclockwise & clockwise & 0.80 (0.38 to 1.70$)$ & 0.57 \\
\hline Aortic root wedging (mm) & 37.78 & 47.19 & $0.92(0.58$ to 1.46$)$ & 0.72 \\
\hline Frontal aortic root tilt angle $\left(^{\circ}\right)$ & 42.20 & 53.73 & 1.01 (0.67 to 1.51$)$ & 0.97 \\
\hline $\begin{array}{l}\text { Left ventricle to aortic root angle: three-chamber } \\
\text { plane }\left(^{\circ}\right)\end{array}$ & 122.45 & 133.70 & 0.85 (0.54 to 1.34$)$ & 0.48 \\
\hline Membranous septum height (mm) & 3.18 & 5.20 & 0.91 (0.60 to 1.38$)$ & 0.66 \\
\hline Membranous septum width (mm) & 4.70 & 8.60 & 0.92 (0.56 to 1.51$)$ & 0.76 \\
\hline Membranous septum area (mm) & 11.50 & 34.71 & $0.86(0.51$ to 1.45$)$ & 0.57 \\
\hline Inferior distance (mm) & 1.50 & 4.40 & 0.84 (0.51 to 1.38$)$ & 0.50 \\
\hline Valve implantation depth (mm) & 3.83 & 7.40 & 1.62 (1.01 to 2.61) & 0.05 \\
\hline$\Delta$ Inferior distance to implantation depth $(\mathrm{mm})$ & -5.06 & -0.59 & 0.61 (0.38 to 0.99$)$ & 0.05 \\
\hline Valve oversizing (\%) & 4.23 & 19.55 & 1.19 (0.73 to 1.94$)$ & 0.48 \\
\hline $\begin{array}{l}\text { Self/mechanical-expandable versus balloon- } \\
\text { expandable valves }\end{array}$ & Balloon & Self/mechanical & 2.35 (1.13 to 4.87$)$ & 0.02 \\
\hline \multicolumn{5}{|l|}{ Valve type } \\
\hline Boston & Edwards & Boston & 4.51 (1.75 to 11.56$)$ & 0.01 \\
\hline Medtronic & Edwards & Medtronic & 1.73 (0.78 to 3.87 ) & \\
\hline
\end{tabular}

OR and 95\% Cl estimated from logistic regression. OR for a comparison of the IQR (Q3 vs Q1). P value for the Wald test.

Q1, quartile 1 (25th percentile); Q3, quartile 3 (75th percentile); TAVI, transcatheter aortic valve implantation; Inferior distance, virtual basal ring to muscular ventricular septum height; $\Delta$ inferior distance to implantation depth, difference between inferior distance and valve implantation depth.

mechanically or self-expanding valves $(\mathrm{OR}=2.35$; CI 1.13 to 4.87). This was more marked when comparing only the mechanically expanding Lotus valve to the balloon-expanding valve $(\mathrm{OR}=4.51$; CI 1.75 to 11.56). Inclusion of only the balloon- and self-expanding valves, and hence exclusion of the Lotus valve, revealed that insertion of the Lotus valve was driving the procedural association with PPI following TAVI for the entire cohort (table 5). No material differences were observed in sensitivity analyses restricted to patients free of atrial fibrillation at baseline (data not shown). The frequency of all six types of valves used and their association with conduction damage are shown in table 6 .

\section{CT and fluoroscopic angiographic measurements}

Procedural characteristics as assessed by both CT and fluoroscopic angiography, and their relationship to both PPI and new-onset LBBB, are reported in tables 2-5. The height of the membranous septum, as well as its inferior distance, demonstrated no association with the occurrence of conduction damage. In contrast, both the depth of implantation and the $\Delta$ inferior distance to implantation depth were associated with PPI $(\mathrm{OR}=1.62$, CI 1.01 to $2.61 ; \mathrm{OR}=0.61$, CI 0.38 to 0.99 , respectively) and new-onset $\mathrm{LBBB}(\mathrm{OR}=1.82$, CI 1.14 to 2.88 ; $\mathrm{OR}=0.64$, CI 0.41 to 1.01 , respectively). The ORs for the inferior distance and depth of implantation were unchanged with mutual adjustment, and no correlation was observed between these measures $(\mathrm{rho}=0.02 ; \mathrm{p}=0.80)$. The mean depth of implantation below the virtual basal plane was $6.1 \pm 2.2 \mathrm{~mm}$ for the balloon-expanding valves, $5.4 \pm 3.2$ $\mathrm{mm}$ for the mechanically expanding valve and $5.4 \pm 2.7$ $\mathrm{mm}$ for the self-expanding valves. Controlling for depth of implantation did not impact the risk of PPI associated with the type of valve, nor vice versa. The degrees of wedging or rotation were neither associated with the occurrence of PPI ( $\mathrm{OR}=0.92$, CI 0.58 to 1.46 ; $\mathrm{OR}=0.92$, CI 0.61 to 1.39 , respectively) nor new-onset $\mathrm{LBBB}(\mathrm{OR}=0.68$, CI 0.43 to $1.08 ; \mathrm{OR}=1.03$, CI 0.70 to 1.52 , respectively). A separate analysis of the 26 patients who received PPI for high-degree atrioventricular block revealed similar associations for the overall group requiring PPI (regardless of indication), with the exceptions of depth of implantation or the $\Delta$ inferior distance to implantation depth (table 7).

\section{DISCUSSION}

Our study shows that preprocedural RBBB, the type of valve inserted, its depth of implantation and the $\Delta$ inferior distance to implantation depth were associated with both requirements for PPI and new-onset LBBB following TAVI. In contrast to prior studies, ${ }^{78}$ no gross anatomical variable in isolation demonstrated any association with conduction damage. While the variables in this investigation found to be associated with conduction damage have been demonstrated previously, ${ }^{7818-22}$ our 


\begin{tabular}{|c|c|c|c|c|}
\hline & Q1/reference & Q3/risk & OR (95\% Cl) & $P$ value \\
\hline Preprocedural QRS duration* (ms) & 92 & 126 & 0.40 (0.18 to 0.87$)$ & 0.01 \\
\hline Preprocedural RBBB & No & Yes & 0.27 (0.08 to 0.92) & 0.04 \\
\hline Aortic root rotation & 2.05 & 19.25 & 1.03 (0.70 to 1.52$)$ & 0.88 \\
\hline Aortic root qualification & Central/counterclockwise & Clockwise & 1.06 (0.53 to 2.12$)$ & 0.86 \\
\hline Aortic root wedging & 37.78 & 47.19 & 0.68 (0.43 to 1.08) & 0.10 \\
\hline Frontal aortic root tilt angle & 42.20 & 53.73 & $0.92(0.62$ to 1.35$)$ & 0.66 \\
\hline $\begin{array}{l}\text { Left ventricle to aortic root angle: three-chamber } \\
\text { plane }\end{array}$ & 122.45 & 133.70 & 0.92 (0.60 to 1.42) & 0.71 \\
\hline Membranous septum height & 3.18 & 5.20 & 1.07 (0.72 to 1.58$)$ & 0.75 \\
\hline Membranous septum width & 4.70 & 8.60 & 1.30 (0.82 to 2.06$)$ & 0.27 \\
\hline Membranous septum area & 11.50 & 34.71 & 1.31 (0.82 to 2.11) & 0.26 \\
\hline Inferior distance & 1.50 & 4.40 & 1.04 (0.66 to 1.65$)$ & 0.87 \\
\hline Valve implantation depth & 3.83 & 7.40 & 1.82 (1.14 to 2.88) & 0.01 \\
\hline$\Delta$ Inferior distance to implantation depth & -5.06 & -0.59 & 0.64 (0.41 to 1.01) & 0.06 \\
\hline Valve oversizing & 4.23 & 19.55 & 1.24 (0.78 to 1.97$)$ & 0.35 \\
\hline $\begin{array}{l}\text { Self/mechanical-expandable versus balloon- } \\
\text { expandable valves }\end{array}$ & Balloon & Self/mechanical & $2.12(1.07$ to 4.20$)$ & 0.03 \\
\hline
\end{tabular}

Valve type

\begin{tabular}{|c|c|c|c|c|}
\hline Boston Scientific & Edwards & Boston Scientific & 3.53 (1.42 to 8.80$)$ & 0.02 \\
\hline Medtronic & Edwards & Medtronic & 1.70 (0.80 to 3.58$)$ & \\
\hline
\end{tabular}

OR and $95 \% \mathrm{Cl}$ estimated from logistic regression. OR for a comparison of the IQR (Q3 vs Q1). P value for the Wald test for total model terms.

${ }^{*}$ Model includes restricted cubic spline term with three knots placed at the 10th, 50th and 90th percentiles.

LBBB, left bundle branch block; Q1, quartile 1 (25th percentile); Q3, quartile 3 (75th percentile); RBBB, right bundle branch block; TAVI, transcatheter aortic valve implantation; Inferior distance, virtual basal ring to muscular ventricular septum height; $\Delta$ inferior distance to implantation depth, difference between inferior distance and valve implantation depth.

current study adds clarity by using anatomically accurate methods. These have demonstrated that neither variation in the height of the membranous septum nor the distance between the crest of the muscular ventricular septum and the virtual basal ring, which we deemed the inferior distance, nor the extent of aortic root wedging and rotation, impact on the risk of conduction damage following TAVI.

These findings appear surprising, as it is intuitive to presume that the distance between the virtual basal ring and the crest of the muscular ventricular septum, which we described as the inferior distance, thought to reflect the presumed vulnerability of the atrioventricular conduction axis, should be an important consideration when valves are implanted in the aortic position. ${ }^{23}$ Measurements of the height of the membranous septum in non-anatomical fashion had suggested a relationship to the occurrence of conduction damage. ${ }^{78}$ Subsequent studies have raised questions regarding this association, ${ }^{10}{ }^{11}$ with the current study confirming the lack of relationship. While the average height of the membranous septum, when measured in an anatomically accurate fashion, is approximately $4-7 \mathrm{~mm}$, the distance between the crest of the muscular septum and the virtual basal plane, is, on average, no more than $2-3 \mathrm{~mm}$, consistent with the averaged measurement observed in our study. Considering the average implantation depth of $5.7 \mathrm{~mm}$ below the virtual basal ring, four-fifths of the valves implanted in our patients had impinged on the crest of the muscular ventricular septum. This fact contrasts with the relatively lower observed incidence of either PPI (20.5\%) or new-onset LBBB (23.5\%). It implies that other factors may influence the occurrence of the conduction disturbances. These could include the proportion of the atrioventricular conduction axis and proximal left bundle branch directly covered by the implanted valve, the depth of the atrioventricular conduction axis from the left ventricular endocardial surface, the extent of bulging of the muscular septum just beneath the membranous septum, and the presence and distribution of calcification. This is in addition to the fact that the origin of the left bundle branch often resides in a more superior position compared with the atrioventricular conduction axis (figure 2). ${ }^{24}$ Of the one-fifth of patients in whom the implanted valve did not reach the crest of the muscular ventricular septum, $10 \%$ subsequently underwent PPI, and another 16\% developed new-onset LBBB without high-degree atrioventricular block. It is intriguing that while the origin of the left bundle branch often resides above the plane of the virtual basal ring, ${ }^{102} 78 \%$ of 
Table 5 CT and procedural characteristics and their association with permanent pacemaker insertion following TAVI in patients (Lotus valve excluded)

\begin{tabular}{|c|c|c|c|c|}
\hline & Q1/reference & Q3/risk & OR $(95 \% \mathrm{Cl})$ & p-value \\
\hline Preprocedural QRS duration* $(\mathrm{ms})$ & 92 & 126 & $1.72(1.02$ to 2.90$)$ & 0.04 \\
\hline Preprocedural RBBB & No & Yes & $7.43(2.97$ to 18.58$)$ & $<0.001$ \\
\hline Aortic root rotation $\left(^{\circ}\right)$ & 2.30 & 18.60 & $0.92(0.58$ to 1.46$)$ & 0.71 \\
\hline Aortic root qualification & Central/counterclockwise & Clockwise & 0.75 (0.31 to 1.81$)$ & 0.52 \\
\hline Aortic root wedging (mm) & 37.50 & 47.30 & 0.97 (0.57 to 1.65$)$ & 0.90 \\
\hline Frontal aortic root tilt angle $\left({ }^{\circ}\right)$ & 41.85 & 54.40 & 0.91 (0.55 to 1.51$)$ & 0.73 \\
\hline Left ventricle to aortic root angle: three-chamber plane ${ }^{\star}\left({ }^{\circ}\right)$ & 122.55 & 134.30 & $1.2(0.7$ to 2.06$)$ & 0.51 \\
\hline Membranous septum height (mm) & 3.10 & 5.10 & 0.94 (0.58 to 1.51$)$ & 0.79 \\
\hline Membranous septum width (mm) & 4.75 & 8.60 & 1.01 (0.57 to 1.77$)$ & 0.98 \\
\hline Membranous septum area (mm) & 10.83 & 34.27 & $0.92(0.5$ to 1.7$)$ & 0.79 \\
\hline Inferior distance (mm) & 1.40 & 4.20 & 0.88 (0.5 to 1.53$)$ & 0.64 \\
\hline Valve implantation depth (mm) & 4.03 & 7.36 & 1.46 (0.85 to 2.52$)$ & 0.17 \\
\hline$\Delta$ Inferior distance to implantation depth $(\mathrm{mm})$ & -5.14 & -0.67 & 0.69 (0.39 to 1.2$)$ & 0.18 \\
\hline Valve oversizing (\%) & 4.65 & 21.25 & 1.68 (0.93 to 3.04$)$ & 0.09 \\
\hline Self-expandable versus balloon-expandable valves & Balloon & Self & 1.73 (0.78 to 3.87$)$ & 0.18 \\
\hline
\end{tabular}

Inferior distance, virtual basal ring to muscular ventricular septum height; $\Delta$ inferior distance to implantation depth, difference between inferior distance and valve implantation depth.

OR and 95\% Cl estimated from logistic regression. OR for a comparison of the IQR (Q3 vs Q1). P value for the Wald test.

*Model includes restricted cubic spline term with three knots placed at the 10th, 50th and 90th percentiles.

Q1, quartile 1 (25th percentile); Q3, quartile 3 (75th percentile); RBBB, right bundle branch block; TAVI, transcatheter aortic valve implantation.

cases in one study, ${ }^{24}$ the incidence of new-onset LBBB in our patients is well below the incidence of the overlap of implanted prosthesis relative to the origin of the left bundle branch.

While neither the height of the membranous septum nor its inferior distance demonstrated a relationship with conduction damage following TAVI, we have confirmed the association with depth of implantation. Our methods also demonstrate the importance of the $\Delta$ inferior distance to implantation depth measurement. Neither of these associations persisted when only patients receiving PPI for the indication of high-degree atrioventricular block were considered. Nor did they persist when we excluded the mechanically expanding Lotus valve from our analysis. Our data support that the association of conduction damage following TAVI with the $\Delta$ inferior distance to implantation depth measurement is primarily driven by the depth of implantation, and not the measurement of the inferior distance. Variation in gross anatomical features of the aortic root and the membranous septum, therefore, are not the major determinants of conduction damage. The conduction damage observed, furthermore, cannot be ascribed exclusively to the leading edge of the prosthesis impinging on the atrioventricular conduction axis. Impingement over a significant distance may be required to produce damage (figure 3 ). Both the increased occurrence of significant conduction damage following implantation of mechanically or self-expanding valves, which on average were implanted to a shallower depth than the balloon-expanding valves, and the lack of association of procedural characteristics (including the depth of implantation) with PPI following TAVI when excluding the mechanically expanding valve, further support the premise that it may be the radial forces on the conduction axis during implantation, more than the actual depth of implantation, regardless of gross

Table 6 Valve type association with PPI and LBBB following TAVI

\begin{tabular}{llll}
\hline Reference valve & Comparison valve & PPI OR (95\% CI) & LBBB OR (95\% CI) \\
\hline Sapien $3(n=83)$ & Sapien XT $(n=11)$ & $0.54(0.06$ to 4.57$)$ & $1.20(0.23$ to 6.18$)$ \\
Sapien 3 & CoreValve $(n=10)$ & $2.02(0.47$ to 8.63$)$ & $1.20(0.23$ to 6.18$)$ \\
Sapien 3 & Evolut $R(n=44)$ & $1.43(0.55$ to 3.66$)$ & $1.43(0.55$ to 3.66$)$ \\
Sapien 3 & Evolut Pro $(n=22)$ & $1.20(0.35$ to 4.11$)$ & $3.08(1.08$ to 8.80$)$ \\
Sapien 3 & Lotus $(n=30)$ & $3.12(1.21$ to 8.06$)$ & $4.12(1.62$ to 10.48$)$ \\
\hline
\end{tabular}

LBBB, left bundle branch block;PPI, permanent pacemaker insertion; TAVI, transcutaneous aortic valve implantation. 
Table 7 CT and procedural characteristics and their association with permanent pacemaker insertion for high-degree atrioventricular block following TAVI.

\begin{tabular}{|c|c|c|c|c|}
\hline & Q1/reference & Q3/risk & OR $(95 \% \mathrm{Cl})$ & $P$ value \\
\hline Aortic root rotation $\left({ }^{\circ}\right)$ & 2.05 & 19.25 & $1.10(0.67$ to 1.78$)$ & 0.71 \\
\hline Aortic root qualification & Central/counterclockwise & Clockwise & 1.01 (0.42 to 2.42$)$ & 0.98 \\
\hline Aortic root wedging (mm) & 37.78 & 47.19 & 0.89 (0.51 to 1.56$)$ & 0.68 \\
\hline Frontal aortic root tilt angle $\left({ }^{\circ}\right)$ & 42.2 & 53.73 & $1.02(0.62$ to 1.66$)$ & 0.95 \\
\hline $\begin{array}{l}\text { Left ventricle to aortic root angle: three- } \\
\text { chamber plane }\left({ }^{\circ}\right)\end{array}$ & 122.45 & 133.7 & 0.65 (0.36 to 1.17$)$ & 0.15 \\
\hline Membranous septum height (mm) & 3.18 & 5.2 & 0.90 (0.55 to 1.48$)$ & 0.68 \\
\hline Membranous septum width (mm) & 4.7 & 8.6 & 1.07 (0.60 to 1.94$)$ & 0.81 \\
\hline Membranous septum area (mm) & 11.5 & 34.71 & $0.93(0.50$ to 1.73$)$ & 0.82 \\
\hline Inferior distance $(\mathrm{mm})$ & 1.5 & 4.4 & 0.69 (0.37 to 1.28$)$ & 0.24 \\
\hline Valve implantation depth (mm) & 3.83 & 7.4 & 1.47 (0.82 to 2.62$)$ & 0.19 \\
\hline $\begin{array}{l}\Delta \text { Inferior distance to implantation depth } \\
(\mathrm{mm})\end{array}$ & -5.06 & -0.59 & 0.59 (0.33 to 1.06$)$ & 0.08 \\
\hline Valve oversizing (\%) & 4.23 & 19.55 & 1.14 (0.64 to 2.04$)$ & 0.66 \\
\hline $\begin{array}{l}\text { Self/mechanical-expandable versus } \\
\text { balloon-expandable valves }\end{array}$ & Balloon & Self/mechanical & 2.97 (1.18 to 7.46$)$ & 0.02 \\
\hline \multicolumn{5}{|l|}{ Valve type } \\
\hline Boston & Edwards & Boston & 6.89 (2.30 to 20.66$)$ & $<0.01$ \\
\hline Medtronic & Edwards & Medtronic & $1.82(0.64$ to 5.16$)$ & \\
\hline
\end{tabular}

OR and $95 \% \mathrm{Cl}$ estimated from logistic regression. OR for a comparison of the IQR (Q3 vs Q1).

$P$ value for the Wald test.

Q1, quartile 1 (25th percentile); Q3, quartile 3 (75th percentile); TAVI, transcatheter aortic valve implantation; Inferior distance, virtual basal ring to muscular ventricular septum height; $\Delta$ inferior distance to implantation depth, difference between inferior distance and valve implantation depth.

anatomical features, which increases the risk for conduction damage. The lack of an association with oversizing of the prosthesis in our study suggests that the force of expansion, rather than the diameter of expansion, imparts greatest risk. This hypothesis is supported by patient-specific computer simulations, which showed the degree of pressure, and the area of contact pressure over the presumed location of the atrioventricular conduction axis, rather than coverage of this area or deeper implantation, imparted the greatest risk of conduction damage following insertion of self-expanding valves. ${ }^{25}$

The lack of any association with variation in the rotational position of the aortic root was an unexpected finding. This may suggest that the position and
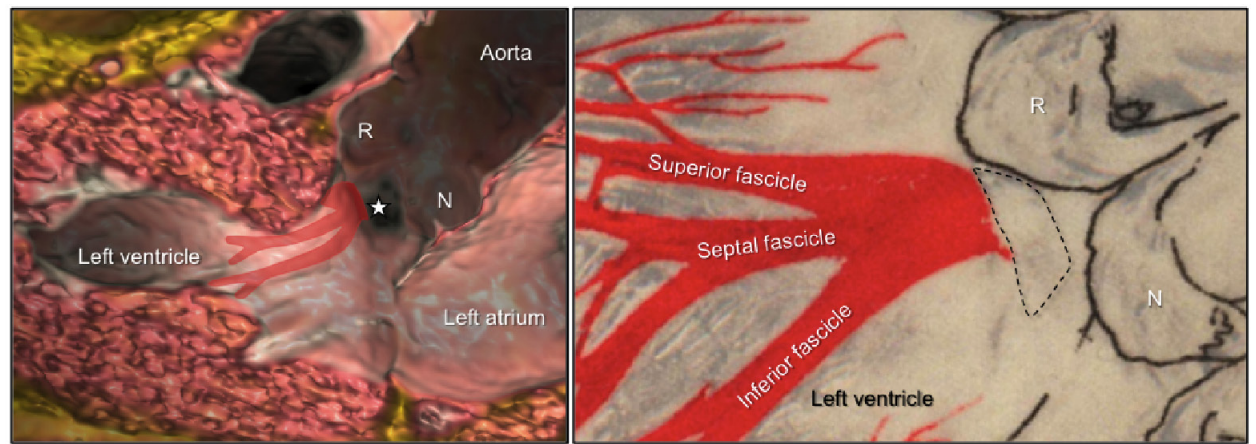

Figure 2 Membranous septum and the proximal left bundle branch. The left panel shows a virtual dissection reconstructed similar to the echocardiographic parasternal long-axis image. The white star indicates the membranous septum. With known knowledge (right panel) concerning the location of the left bundle branch (RED) ramifying from the branching portion of the bundle of $\mathrm{His}^{24}{ }^{26}$ in the antero-inferior margin of the membranous septum (black-dotted area, superimposed on the original image of Tawara ${ }^{26}$ ), such combined images help the interventionists appreciate the danger zone during transcatheter aortic valve implantation. Note that, when described in attitudinally appropriate terminology, the so-called 'anterior' and 'posterior' fascicles of the left bundle are more appropriate referred to as the superior and inferior fascicles respectively. ${ }^{27} \mathrm{R}$, right coronary aortic sinus; N, non-coronary aortic sinus. 

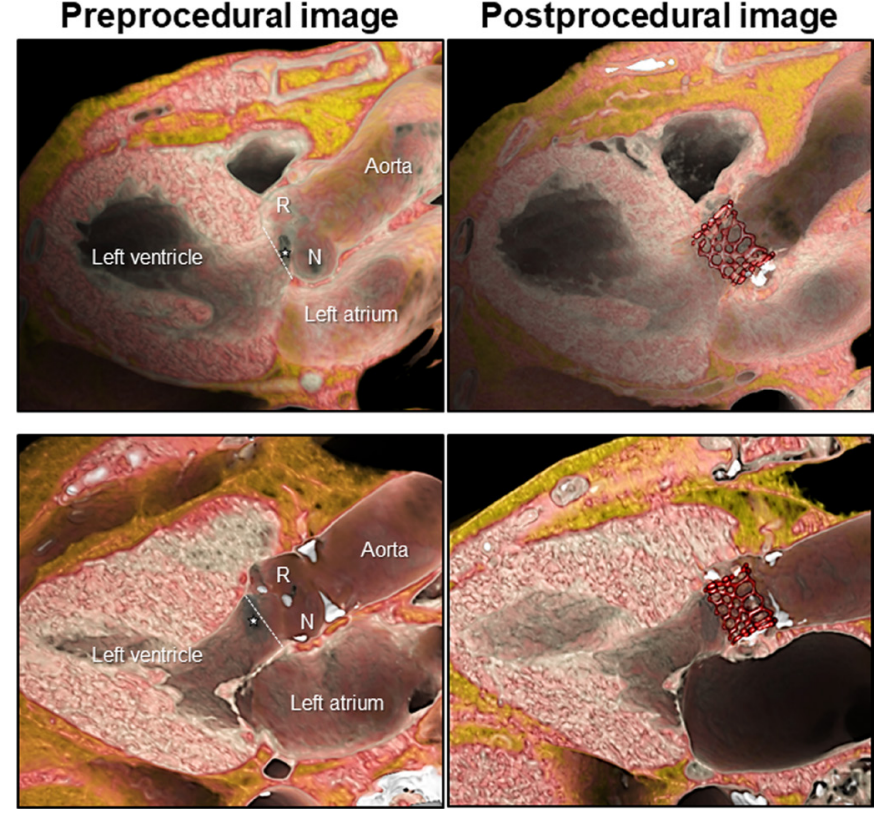

Figure 3 Representative cases with and without conduction damage after transcatheter aortic valve implantation superior panels demonstrate a case with left bundle branch block after implantation of Sapien 3. The virtual basal ring plane (white-dotted line) is located inferior to the membranous septum (white star), and the valve is implanted relatively deeper to the virtual basal ring. Inferior panels demonstrate a case without conduction damage after implantation of Sapien 3. The virtual basal ring plane (white-dotted line) is located superior to the membranous septum (white star), and the valve is implanted approximately at the level of the virtual basal ring. Significant distance is noted between the inferior edge of the valve and the crest of the muscular ventricular septum, where the proximal left bundle branch ramifies from the branching portion of the bundle of His (compare to figure 2). R, right coronary aortic sinus; $N$, non-coronary aortic sinus.

relationship of the atrioventricular conduction axis to the aortic root as well as its length does not vary with the variation observed in the width of the central fibrous body which itself relates to the rotational position of the aortic root. ${ }^{11}$ Since the atrioventricular conduction axis commonly resides angulated relative to the plane of the virtual basal ring with the anterior part located superiorly and the posterior part located inferiorly, ${ }^{24}$ it is intuitive that deep implantation increases the extent of the conduction axis impinged on by the valve. Given the relatively small cohort of self- and mechanically expanding valves, this variation deserves further investigation in considering the impact of contact pressure. ${ }^{25}$ The variation in the breadth of the central fibrous body and the fibrous versus muscular support of the aortic root, nonetheless, may additionally impact the structural integrity of the aortic root before and after TAVI with consequent effects on bioprosthetic durability as well as the occurrence of paravalvar leak.

Our study highlights that the role of anatomical variability in the occurrence and progression of conduction damage remains unclear. Furthermore, although gross or macroscopic anatomical variability may not impact the occurrence of these adverse outcomes, the role of microscopic anatomical variability remains to be seen. A histological study assessing the course of the atrioventricular bundle over the crest of the muscular ventricular septum reported that, in $50 \%$, the axis was located predominately to the right, with $30 \%$ having a leftward bundle and $20 \%$ having a superficial bundle positioned just below the endocardium. ${ }^{24}$ Future studies are required to assess the impact of this variation on the risk for conduction damage following TAVI.

\section{Limitations}

Our study carries the inherent limitations of its retrospective design, with a relatively small cohort and low occurrence of assessed outcomes. Multiple types of bioprosthetic valves were implanted, and we excluded a high proportion of patients. The exclusion of patients with significant renal disease who underwent a low-contrast CT study makes our assessed cohort not generalisable to the global population undergoing TAVI. The extent and location of calcification within the aortic root and the landing zone for the valve were not assessed in this study. It is also the case that three-dimensional structures were assessed in two-dimensional fashion. The membranous septum often does not reside in a plane parallel to the virtual basal ring. ${ }^{1024}$ Measurement of its mid-portion, therefore, may not be representative of the shallowest or deepest aspect of its inferior margin.

\section{CONCLUSION}

We have shown that gross anatomical variations of the aortic root and its underlying support, including the dimensions of the membranous septum, are not associated with the occurrence of either PPI or new-onset LBBB. Preprocedural RBBB, the type of valve, specifically the mechanically expanding valve, the depth of implantation and $\Delta$ inferior distance to implantation depth were all associated with these adverse outcomes. It is the depth and radial force of implantation, regardless of gross anatomical variability, which is associated with the occurrence of conduction damage.

Contributors JTT originated the concept of the work, performed the data gathering, wrote the original manuscript and made significant revisions leading to the final manuscript. SM helped originate the concept of the work and provided significant revisions leading to the final manuscript. RA provided significant revisions leading to the final version of the manuscript. MT supervised the data gathering and made significant revisions to the final manuscript. NJO performed the statistical analysis and made revisions to the statistical and results sections of the manuscript. VTT helped with some of the data gathering. JKC helped originate the concept of the work and provided revisions leading to the final manuscript. DJK provided significant revisions leading to the final manuscript. WM provided significant revisions leading to the final manuscript.

Funding The authors have not declared a specific grant for this research from any funding agency in the public, commercial or not-for-profit sectors.

Competing interests DJK is on the scientific advisory board for and a consultant for Boston Scientific Corporation.

Patient consent for publication Not required 
Provenance and peer review Not commissioned; externally peer reviewed.

Open access This is an open access article distributed in accordance with the Creative Commons Attribution Non Commercial (CC BY-NC 4.0) license, which permits others to distribute, remix, adapt, build upon this work non-commercially, and license their derivative works on different terms, provided the original work is properly cited, appropriate credit is given, any changes made indicated, and the use is non-commercial. See: http://creativecommons.org/licenses/by-nc/4.0/.

\section{REFERENCES}

1. van Rosendael PJ, Delgado V, Bax JJ. Pacemaker implantation rate after transcatheter aortic valve implantation with early and new-generation devices: a systematic review. Eur Heart $J$ 2018;39:2003-13.

2. Auffret V, Puri R, Urena M, et al. Conduction disturbances after transcatheter aortic valve replacement: current status and future perspectives. Circulation 2017;136:1049-69.

3. Leon MB, Smith CR, Mack MJ, et al. Transcatheter or surgical aortic-valve replacement in intermediate-risk patients. N Engl J Med 2016;374:1609-20.

4. Nishimura RA, Otto CM, Bonow RO, et al. 3rd, Fleisher La, Jneid $\mathrm{H}$, Mack MJ, McLeod CJ, O'Gara Pt, Rigolin VH, Sundt Tm, 3rd, Thompson A. 2017 AHA/ACC focused update of the 2014 AHAVACC guideline for the management of patients with valvular heart disease: a report of the American College of Cardiology/American Heart Association Task Force on clinical practice guidelines. J Am Coll Cardiol 2017;70:252-89.

5. Reardon MJ, Van Mieghem NM, Popma JJ, et al. Surgical or transcatheter aortic-valve replacement in intermediate-risk patients. N Engl J Med 2017;376:1321-31.

6. Achenbach S, Delgado V, Hausleiter J, et al. SCCT expert consensus document on computed tomography imaging before transcatheter aortic valve implantation (TAVI)/transcatheter aortic valve replacement (TAVR). J Cardiovasc Comput Tomogr 2012;6:366-80.

7. Maeno $\mathrm{Y}$, Abramowitz $\mathrm{Y}, \mathrm{Kawamori} \mathrm{H}$, et al. A highly predictive risk model for pacemaker implantation after TAVR. JACC: Cardiovascular Imaging 2017;10:1139-47.

8. Hamdan A, Guetta V, Klempfner R, et al. Inverse relationship between membranous septal length and the risk of atrioventricular block in patients undergoing transcatheter aortic valve implantation. JACC Cardiovasc Interv 2015;8:1218-28.

9. Tretter JT, Spicer DE, Mori S, et al. The significance of the Interleaflet triangles in determining the morphology of congenitally abnormal aortic valves: implications for noninvasive imaging and surgical management. J Am Soc Echocardiogr 2016;29:1131-43.

10. Mori S, Tretter JT, Toba T, et al. Relationship between the membranous septum and the virtual basal ring of the aortic root in candidates for transcatheter implantation of the aortic valve. Clin. Anat. 2018;31:525-34.

11. Tretter JT, Mori S, Saremi F, et al. Variations in rotation of the aortic root and membranous septum with implications for transcatheter valve implantation. Heart 2018;104:999-1005.
12. Mori S, Anderson RH, Takaya T, et al. The association between wedging of the aorta and cardiac structural anatomy as revealed using multidetector-row computed tomography. J Anat 2017;231:110-20.

13. Truong VT, Choo J, McCoy L, et al. Low-volume contrast CT angiography via pulmonary artery injection for measurement of aortic annulus in patients undergoing transcatheter aortic valve replacement. J Invasive Cardiol 2017;29:181-6.

14. Feldman TE, Reardon MJ, Rajagopal V, et al. Effect of mechanically expanded vs self-expanding transcatheter aortic valve replacement on mortality and major adverse clinical events in high-risk patients with aortic stenosis. JAMA 2018;319:27-37.

15. Kim W-K, Moellmann H, Rolf A, et al. TCT-749 comparison of area and perimeter derived effective annulus diameter with direct intraoperative sizing. J Am Coll Cardiol 2013;62.

16. FE H.J. rms: Regression Mod Strategies. R package version 5.1-2. 2018.

17. Team RC. R: a language and environment for statistical computing. Vienna, Austria: R Foundation for Statistical Computing, 2018.

18. Chamandi C, Barbanti M, Munoz-Garcia A, et al. Long-Term Outcomes in Patients With New Permanent Pacemaker Implantation Following Transcatheter Aortic Valve Replacement. JACC CardiovasC Interv 2018;11:301-10.

19. Fadahunsi OO, Olowoyeye A, Ukaigwe A, et al. Predictors, and outcomes of permanent pacemaker implantation following transcatheter aortic valve replacement: analysis from the U.S. Society of thoracic Surgeons/American College of cardiology TVT registry. JACC Cardiovasc Interv 2016;9:2189-99.

20. Husser O, Pellegrini C, Kessler T, et al. Predictors of permanent pacemaker implantations and new-onset conduction abnormalities with the SAPIEN 3 Balloon-Expandable transcatheter heart valve. JACC Cardiovasc Interv 2016;9:244-54.

21. Mauri V, Reimann A, Stern D, et al. Predictors of permanent pacemaker implantation after transcatheter aortic valve replacement with the SAPIEN 3. JACC 2016;9:2200-9.

22. Siontis GCM, Jüni $P$, Pilgrim $T$, et al. Predictors of permanent pacemaker implantation in patients with severe aortic stenosis undergoing TAVR. J Am Coll Cardiol 2014;64:129-40.

23. Piazza Nicoló, de Jaegere P, Schultz C, et al. Anatomy of the aortic valvar complex and its implications for transcatheter implantation of the aortic valve. Circ Cardiovasc Interv 2008;1:74-81.

24. Kawashima T, Sato F. Visualizing anatomical evidences on atrioventricular conduction system for TAVI. Int J Cardiol 2014;174:1-6.

25. Rocatello G, El Faquir N, De Santis G, et al. Patient-specific computer simulation to elucidate the role of contact pressure in the development of new conduction abnormalities after Catheter-Based implantation of a self-expanding aortic valve. Circ Cardiovasc Interv 2018;11:e005344.

26. Tawara S. Das Reizleitungssystem des Saugetierherzens: Eine anatomisch-histologische studie uber das Atrioventrikularbundel und die Purkinjeschen Faden. Jena, Germany: Gustav Fischer, 1906: 9-70114-156.

27. Mori S, Spicer DE, Anderson RH. Revisiting the anatomy of the living heart. Circ J 2016;80:24-33. 